\title{
Theoretical and numerical aspects of stochastic nonlinear Schrödinger equations
}

\author{
Anne de Bouard* \\ Arnaud Debussche ${ }^{\dagger}$ \\ Laurent Di Menza*
}

\begin{abstract}
We describe several results obtained recently on stochastic non linear Schrödinger equations. We show that under suitable smoothness assumptions on the noise, the nonlinear Schrödinger perturbed by an additive or multiplicative noise is well posed under similar assumptions on the nonlinear term as in the deterministic theory. Then, we restrict our attention to the case of a focusing nonlinearity with critical or supercritical exponent. If the noise is additive, smooth in space and non degenerate, we prove that any initial data gives birth to a singular solution ; thus the noise changes the qualitative behavior since, as is well known, in the deterministic case only a restricted class of initial data give a solution which blows up. We also present numerical experiments which indicate that, on the contrary, a multiplicative white noise seems to prevent blow up. We finally give a convergence result for the numerical scheme used in these simulations.
\end{abstract}

\section{Introduction}

The nonlinear Schrödinger (NLS) equation describes the propagation of waves in media with both nonlinear and dispersive response. This model is used in various fields such as hydrodynamics, plasma physics, nonlinear optics, or molecular biology. This equation has localized solutions, called soliton, which are particularly robust and propagate without changing form. More and more attention has been paid recently to the influence of a noise on the propagation of this soliton solution. The dynamics of optical pulses in fibers with randomly varying, birefringence may e.g. be governed by a system of coupled NLS equations with random coefficients (see [10]). A one dimensional NLS equation with an additive space time white noise is considered in [8], for which a method is proposed to calculate the probability distribution of soliton parameters. The equation reads

$$
i \partial_{t} \psi-\left(\partial_{x}^{2} \psi+2|\psi|^{2} \psi\right)=\xi, \quad x \in \mathbb{R}, \quad t \geq 0,
$$

MSC 2000: 35Q55, 60H15, 76B15, 76M35, 65M06, 65M30, 35L67.

Keywords : stochastic partial differential equations, nonlinear Schrödinger equation, blow-up, white noise, finite difference scheme, mesh refinement. 
and $\xi$ is a space-time white noise. A nonlinear Schrödinger equation with multiplicative noise has also been proposed as a model of energy transfer in a monolayer molecular aggregate in the presence of thermal fluctuations (see [1]). In this case, the noise arises as a real valued potential, and the equation, which may be written as

$$
i \partial_{t} \psi-\left(\Delta \psi+|\psi|^{2} \psi\right)=\eta \psi, \quad x \in \mathbb{R}^{2}, \quad t \geq 0,
$$

must preserve the $L^{2}$ norm of the solution $\psi$ as $t$ varies, since $|\psi|^{2}$ stands here for a (spatial) probability density. It follows that the product arising in the right hand side of (1.2) is necessarily a Stratonovitch product.

In section 2, we briefly recall the definition of a white noise and the construction of the stochastic integral. In particular, we will see that a space-time white noise has very irregular paths. Since the Schödinger equation has little smoothing effects, it seems difficult to treat such noises. In our theoretical study, we thus consider noises which are white in time but are correlated in space. We give in section 3 various existence and uniqueness results for equations (1.1) and (1.2). In section 4, we study the influence of an additive noise on finite time blow-up of solutions in the focusing case $(\lambda=+1)$ and with $\sigma$ critical or supercritical. We show that the noise has a strong effect, when it is spatially smooth : any solution blows up in finite time whereas in the deterministic case, only a restricted class of initial data give birth to blowing up solutions. We use in section 5 numerical simulations to strengthen the theoretical results of section 4; we first observe that any solution blows up with probability one and that our results seem to be valid also with a smooth multiplicative noise, at least for supercritical nonlinearities. We then verify numerically the conjecture that a multiplicative space-time white noise may prevent blow-up; such a behavior is totally different from the smooth noise case. Finally, in section 6 , we state a convergence result for the semi-discrete version of the scheme that we used in section 5 .

\section{Mathematical description of the noise}

In order to state precisely the equations we will consider in this note, we introduce a probability space $(\Omega, \mathcal{F}, \mathbb{P})$ endowed with a filtration $\left(\mathcal{F}_{t}\right)_{t \geq 0}$. Let us recall that a filtration is an increasing family of $\sigma$-algebra contained in $\mathcal{F}$ and that, roughly speaking, a random variable which is measurable with respect to $\mathcal{F}_{t}$ depends only on the past up to time $t$.

A brownian motion $\beta$ is a gaussian process with independent increments, i.e. a family of random variables indexed by the time $t$ such that, for each $t_{1}, t_{2}, \ldots, t_{n}$, $\left(\beta\left(t_{1}\right), \beta\left(t_{2}\right), \ldots, \beta\left(t_{n}\right)\right)$ is a gaussian vector and, for $t \geq s, \beta(t)-\beta(s)$ and $\beta(s)$ are independent. It is adapted to $\left(\mathcal{F}_{t}\right)_{t \geq 0}$ if for each $t, \beta(t)$ is measurable with respect to $\mathcal{F}_{t}$ and, for $t \geq s, \beta(t)-\beta(s)$ is independent of $\mathcal{F}_{s}$. This means that the increments do not depend on the past.

It is well known that for almost every $\omega \in \Omega$, the trajectory $t \mapsto \beta(t, \omega)$ is continuous, and even $\alpha$-hölder continuous for any $\alpha<1 / 2$, but nowhere differentiable and not of bounded variation.

The white noise in time $\dot{\beta}$ is the distributional derivative of the brownian motion 
with respect to time :

$$
\dot{\beta}=\frac{d \beta}{d t} .
$$

It is delta correlated in the sense that, formally,

$$
\mathbb{E}(\dot{\beta}(t) \dot{\beta}(s))=\delta_{t-s},
$$

where $\delta$ is the Dirac mass. The name "white noise" originates from the fact that the Dirac mass has a constant Fourier transform.

We can similarly define the space-time white noise $\xi$ as the derivative with respect to the space and time variables of a multidimensional brownian motion $B(t, x)$ :

$$
\xi=\frac{\partial^{n+1} B}{\partial t \partial x_{1} \ldots \partial x_{n}} .
$$

As before, $B$ is $\alpha$-hölder continuous for $\alpha<1 / 2$ with respect to $(t, x)$ but is not of bounded variations.

However, in the context of evolutionary partial differential equations, it is convenient to treat the time $t$ and the space $x$ differently and we use another equivalent construction.

We consider a sequence $\left(\beta_{k}\right)_{k \in \mathbb{N}}$ of independent real valued Brownian motions on $\mathbb{R}^{+}$associated to the filtration $\left(\mathcal{F}_{t}\right)_{t \geq 0}$, a hilbertian basis $\left(e_{k}\right)_{k \in \mathbb{N}}$ of $L^{2}\left(\mathbb{R}^{n}\right)$, the space of square integrable complex valued functions, and a bounded linear operator $\Phi$ on $L^{2}\left(\mathbb{R}^{n}\right)$. The process

$$
W(t, x, \omega)=\sum_{k=0}^{\infty} \beta_{k}(t, \omega) \Phi e_{k}(x), \quad t \geq 0, \quad x \in \mathbb{R}^{n}, \quad \omega \in \Omega,
$$

is a Wiener process on the space of square integrable functions on $\mathbb{R}^{n}$, with covariance operator $\Phi \Phi^{*}$. We then set

$$
\xi=\frac{\partial W}{\partial t} .
$$

This defines a complex valued noise which is delta correlated in time and whose space correlation depends on $\Phi$.

Similarly, if we take a hilbertian basis of $L_{\mathbb{R}}^{2}\left(\mathbb{R}^{n}\right)$, the space of square integrable real valued function, instead of $L^{2}\left(\mathbb{R}^{n}\right)$ and if $\Phi$ maps $L_{\mathbb{R}}^{2}\left(\mathbb{R}^{n}\right)$ into itself, we obtain a real valued noise.

Note that if $\Phi$ is defined through a kernel $\mathcal{K}$, which means that for any square integrable function $u$,

$$
\Phi u(x)=\int_{\mathbb{R}^{n}} \mathcal{K}(x, y) u(y) d y,
$$

then the correlation function of the noise is formally given by

$$
\mathbb{E}\left(\frac{\partial W}{\partial t}(t, x) \frac{\partial W}{\partial t}(s, y)\right)=c(x, y) \delta_{t-s}
$$

with

$$
c(x, y)=\int_{\mathbb{R}^{n}} \mathcal{K}(x, z) \mathcal{K}(y, z) d z .
$$


The space-time white is obtained when $\Phi$ is the identity, corresponding to $k(x, y)=$ $c(x, y)=\delta_{x-y}$. However such a noise has very irregular trajectories, $W$ is almost surely continuous with respect to time with values in the negative Sobolev space $H_{l o c}^{s}\left(\mathbb{R}^{n}\right), s<-n / 2$. And we are not able to consider such a rough noise in the context of the NLS equation which has very little smoothing effect. It can be shown that if $\Phi$ is a Hilbert-Schmidt operator from $L^{2}\left(\mathbb{R}^{n}\right)$ to another Hilbert space $\widetilde{H}$ then $W$ is almost surely continuous with respect to time with values in $\widetilde{H}$. We will make such assumptions on $\Phi$.

Once a Wiener process $W$ is given, we define an integral with respect to $W$ :

$$
\int_{0}^{t} \psi(s) d W(s)
$$

Since $W$ is constructed in a Hilbert space, the integrand $\psi(t)$ should be a mapping with values in a space of linear operator on this Hilbert space. As previously mentioned, $W$ is not of bounded variation and the integral cannot be defined as a Stieljes integral. However $W$ has finite quadratic variation and, using probabilistic arguments, this integral can be defined as the limit of the Riemann sums

$$
\sum_{i=1}^{N} \psi\left(t_{i}\right)\left(W\left(t_{i+1}\right)-W\left(t_{i}\right)\right)
$$

where $t_{1}, \ldots, t_{N}$ is a subdivision of $[0, T]$. The integral will make sense as an element of a Hilbert space $K$ provided

$$
\int_{0}^{t}\left\|\Phi^{*} \psi(s)\right\|_{L_{H S}\left(L^{2}\left(\mathbb{R}^{n}\right), K\right)}^{2} d s<\infty
$$

where $L_{H S}\left(L^{2}\left(\mathbb{R}^{n}\right), K\right)$ is the space of Hilbert-Schmidt operators from $L^{2}\left(\mathbb{R}^{n}\right)$ to $K$. In this way, we obtain the Ito integral.

Since $W$ is not of bounded variation, the choice of the point in the Riemann sums has an influence on the resulting integral. If the integrand is evaluated at the mid point, i.e. if $\psi\left(\frac{t_{i}+t_{i+1}}{2}\right)$ is taken instead of $\psi\left(t_{i}\right)$ in the above formula, the Stratonovitch integral is obtained.

\section{Well-posedness results}

In this section, we gather different results on existence and uniqueness for the stochastic NLS equation with additive or multiplicative noise. The latter case is the most difficult to treat, the noise depends on the solution through a Lipschitz function (multiplication by the solution) which is not bounded. This creates difficulties in deriving a-priori estimates. Moreover, we use a contraction argument in spaces of the form $L^{r}\left(0, T ; L^{p}\left(\mathbb{R}^{n}\right)\right)$ and this requires the use of Burkholder inequalities in Banach spaces.

We first rewrite the equations with the notations introduced above. We are given $\sigma>0, \lambda= \pm 1$, and a Wiener process $W$ associated to a linear operator $\Phi$. We then 
consider two types of stochastic NLS equations : an additive one which is written as

$$
i d u-\left(\Delta u+\lambda|u|^{2 \sigma} u\right) d t=d W
$$

and a multiplicative one, written as

$$
i d u-\left(\Delta u+\lambda|u|^{2 \sigma} u\right) d t=u \circ d W
$$

where o stands for a Stratonovitch product in the right hand side of (3.4). We will use the equivalent Ito equation. Defining for $x \in \mathbb{R}^{n}$ the function

$$
F_{\Phi}(x)=\sum_{k=0}^{\infty}\left(\Phi e_{k}(x)\right)^{2}
$$

which does not depend on the basis $\left(e_{k}\right)_{k \in \mathbb{N}}$, this equivalent Ito equation may be written as

$$
i d u-\left(\Delta u+\lambda|u|^{2 \sigma} u\right) d t=u d W-\frac{i}{2} u F_{\Phi} d t .
$$

In equation (3.3), the noise is either real or complex valued. In equation (3.4), the noise is a potential and is real valued.

We have the following result.

Theorem 3.1 i) Let $n \leq 5, \sigma>0$ if $n=1$ or $2,0<\sigma<\frac{2}{n-2}$ if $n=3$ and $\frac{1}{2}<\sigma<\inf \left(\frac{2}{n-2}, \frac{3}{2(n-3)}\right)$ if $n=4$ or 5 . Assume that $\Phi$ is a Hilbert-Schmidt operator from $L^{2}\left(\mathbb{R}^{n}\right)$ into $H^{1}\left(\mathbb{R}^{n}\right)$, and if $n \geq 2$, assume moreover that $\Phi$ is a $\gamma$-radonifying operator from $L^{2}\left(\mathbb{R}^{n}\right)$ into $W^{1, \alpha}\left(\mathbb{R}^{n}\right)$ for some $\alpha>2 n$. Then for any $\mathcal{F}_{0}$-measurable $u_{0}$ with values in $H^{1}\left(\mathbb{R}^{n}\right)$, there is a unique solution starting from $u_{0}$ to equation (3.5) with continuous $H^{1}\left(\mathbb{R}^{n}\right)$ valued paths. Moreover, the existence time $\tau^{*}\left(u_{0}\right)$, which is a stopping time, satisfies :

$$
\tau^{*}\left(u_{0}\right)=+\infty \quad \text { or } \quad \lim _{t / \tau^{*}\left(u_{0}\right)}|u(t)|_{H^{1}\left(\mathbb{R}^{n}\right)}=+\infty \quad \text { a.s. }
$$

The solution is global if moreover $\sigma<2 / n$ or in the case of a defocusing nonlinearity $\lambda=-1$, and if in addition $\Phi$ satisfies some smoothness assumptions.

ii) Let $n \in \mathbb{N}^{*}$ and assume that $0<\sigma<2 / n$ if $n=1$ or $2,0<\sigma<\frac{1}{n-1}$ if $n \geq 3$, and that $\Phi$ is a $\gamma$-radonifying operator from $L^{2}\left(\mathbb{R}^{n}\right)$ into $L^{2+\delta}\left(\mathbb{R}^{n}\right)$ for some $\delta>2(n-1)$. Then one can find a pair $(r, p)$, with $r, p \geq 2$, such that for any $\mathcal{F}_{0}$-measurable initial data with values in $L^{2}\left(\mathbb{R}^{n}\right)$, there is a unique solution to (3.5) with paths in $\mathcal{C}\left(\left[0, T_{0}\right] ; L^{2}\left(\mathbb{R}^{n}\right)\right) \cap L^{r}\left(0, T_{0} ; L^{p}\left(\mathbb{R}^{n}\right)\right)$ where $T_{0}$ is any given positive time.

We recall that a $\gamma$-radonifying operator from a Hilbert space $H$ into a Banach space $B$ is a bounded linear operator $K$ from $H$ into $B$ such that the image by $K$ of the canonical gaussian distribution on $H$ extends to a Borel probability measure on $B$.

The statement on $L^{2}$ solutions in Theorem 3.1 is proved in [3]. Similar techniques are used for $H^{1}$ solutions, details on this aspect are given in [6]. 
The case of an additive noise is much easier to treat. Indeed, we introduce

$$
z(t)=\int_{0}^{t} U(t-s) d W(s)
$$

where $(U(t))_{t \in \mathbb{R}}$ is the group associated to the linear Schrödinger equation, and translate the unknown by setting : $v=u-z$. Since $z$ solves the linear equation

$$
i d z-\Delta z=d W
$$

we are lead to the following random modified Schrödinger equation which can be solved pathwise thanks standard deterministic methods :

$$
i \frac{d v}{d t}-\left(\Delta v+\lambda|v+z|^{2 \sigma}(v+z)\right)=0
$$

The following result is proved in [6].

Theorem 3.2 i) Assume that $0 \leq \sigma<2 /(n-2)$, $\Phi$ is a Hilbert-Schmidt operator from $L^{2}\left(\mathbb{R}^{n}\right)$ into $H^{1}\left(\mathbb{R}^{n}\right)$, and that the initial data is $\mathcal{F}_{0}$-measurable with values in $H^{1}\left(\mathbb{R}^{n}\right)$. Then there exists a unique solution to (3.3) with continuous $H^{1}\left(\mathbb{R}^{n}\right)$ valued paths. This solution is defined on a random interval $\left[0, \tau^{*}\left(u_{0}\right)\right)$, where $\tau^{*}\left(u_{0}\right)$ is a stopping time such that

$$
\tau^{*}\left(u_{0}\right)=+\infty \quad \text { or } \quad \lim _{t / \tau^{*}\left(u_{0}\right)}|u(t)|_{H^{1}\left(\mathbb{R}^{n}\right)}=+\infty \quad \text { a.s. }
$$

ii) If $\sigma<2 / n$ or in the defocusing case $\lambda=-1$, the solution is global: $\tau^{*}\left(u_{0}\right)=+\infty$ for any $u_{0} \in H^{1}\left(\mathbb{R}^{n}\right)$.

iii) Furthermore, if $\sigma<2 / n$, the statement in i) is still valid with $H^{1}\left(\mathbb{R}^{n}\right)$ replaced by $L^{2}\left(\mathbb{R}^{n}\right)^{1}$ and the solution is global.

In Theorem 3.2, the assumptions on the nonlinearity are the same as in the deterministic theory and it seems difficult to weaken them. On the contrary, in Theorem 3.1, additional restrictions are imposed on $\sigma$ compared to the deterministic case. They are due to the impossibility to use Strichartz estimates on the stochastic integral.

It is important to consider both $L^{2}$ and $H^{1}$ solutions. Indeed, the physical models use a space time white noise. It is impossible to treat it with the available techniques, but we think that we should try to lower the space regularity assumptions on the noise as much as we can. Up to now, the $L^{2}$ framework provides minimal assumptions. However, when studying blow-up phenomena, it is natural to work with $H^{1}$ solutions.

\footnotetext{
$T>0$.

${ }^{1}$ except for uniqueness which holds in $L^{\infty}\left(0, T, L^{2}\left(\mathbb{R}^{n}\right)\right) \cap L^{r}\left(0, T, L^{p}\left(\mathbb{R}^{n}\right)\right)$ for some $p$ and any
} 


\section{Blow-up in finite time}

It is well known that for the deterministic focusing NLS equation $(\lambda=+1)$ with $2 / n \leq \sigma<2 /(n-2)$, there exist solutions which develop singularities in finite time (see [9] and reference therein). A natural question is to try to understand the influence of a noise on such phenomena. We show that the presence of a smooth additive noise strongly influences the blow-up. Before stating the results, we define the function spaces

$$
\begin{gathered}
\Sigma=\left\{u \in H^{1}\left(\mathbb{R}^{n}\right), \quad|x| u \in L^{2}\left(\mathbb{R}^{n}\right)\right\}, \\
\Sigma^{k, l}=\left\{u \in H^{k+l}\left(\mathbb{R}^{n}\right), \quad|x|^{l} u \in H^{k}\left(\mathbb{R}^{n}\right)\right\},
\end{gathered}
$$

and $S_{n}$ whose description depends on the space dimension $n$,

$$
S_{1}=\Sigma, \quad S_{2}=\bigcup_{\alpha>0} \Sigma^{\alpha, 1}, \quad S_{n}=\Sigma^{1,2} \quad \text { if } n \geq 3
$$

In all this section we assume that $\lambda=+1$ and $\frac{2}{n} \leq \sigma<\frac{2}{n-2}$.

Theorem 4.1 Assume that the covariance operator is such that $\Phi$ is Hilbert-Schmidt from $L^{2}\left(\mathbb{R}^{n}\right)$ into $S_{n}$, and that $\operatorname{Ker} \Phi^{*}=\{0\}$. Then for any $u_{0} \in \Sigma$ and for any $t>0$, if $u$ is the solution of (3.3) starting from $u_{0}$ given by Theorem 3.2, then we have either

$$
\mathbb{P}\left(\tau^{*}\left(u_{0}\right)<t\right)>0
$$

or

$$
\mathbb{E} \int_{0}^{t}\left(|u|_{H^{1}\left(\mathbb{R}^{n}\right)}^{2}+|u|_{L^{2 \sigma+2}\left(\mathbb{R}^{n}\right)}^{4 \sigma+2}\right) d s=+\infty
$$

We recall that for the deterministic equation, blow-up of solutions is proved only for some initial data. It is even known that there exist initial data which yield global existence. Our result proves that in the presence of noise, any initial data immediately yields a singular solution at least in the weak sense (4.7). All the conclusions of Theorem 4.1 are still valid in the case of a real valued noise under the additional assumption that $u_{0} \in S_{n} \cap H^{s}\left(\mathbb{R}^{n}\right)$ for some $s>n / 2$. If we strengthen the assumptions on $\Phi$, we can prove that the blow-up always occurs with positive probability, i.e. (4.6) always happens.

Theorem 4.2 Assume that $\frac{2}{n}<\sigma<\min \left(\frac{2}{3}, \frac{2}{n-2}\right)$ and that the covariance operator is such that $\Phi$ is Hilbert-Schmidt from $L^{2}\left(\mathbb{R}^{n}\right)$ into $S_{n}$, $\gamma$-radonifying from $L^{2}\left(\mathbb{R}^{n}\right)$ into $L^{4 \sigma+2}\left(\mathbb{R}^{n}\right)$, and bounded from $L^{2}\left(\mathbb{R}^{n}\right)$ into $H^{2}\left(\mathbb{R}^{n}\right) \cap L^{\infty}\left(\mathbb{R}^{n}\right)$, with $\operatorname{Ker} \Phi^{*}=$ $\{0\}$, then for any $u_{0} \in \Sigma$ and for any $t>0$,

$$
\left.\mathbb{P}\left(\tau^{*}\left(u_{0}\right)\right)<t\right)>0 .
$$

We note that the assumptions on $\sigma$ are compatible only when $n \geq 4$. However, we believe that our result is true for more general $\sigma$, this problem will be studied in the future. The idea to prove Theorem 4.1 is first to generalize the deterministic 
method and to prove that blow-up occurs in the sense of (4.6) or (4.7) for some initial data. We define for $u \in \Sigma$ :

$$
V(u)=\int_{\mathbb{R}^{n}}|x|^{2}|u(x)|^{2} d x, \quad G(u)=\operatorname{Im} \int_{\mathbb{R}^{n}}(x . \nabla u) \bar{u} d x,
$$

and

$$
H(u)=\frac{1}{2} \int_{\mathbb{R}^{n}}|\nabla u|^{2} d x-\frac{1}{2 \sigma+2} \int_{\mathbb{R}^{n}}|u|^{2 \sigma+2} d x
$$

and prove that if a solution $u$ is such that

$$
\mathbb{P}\left(\tau^{*}\left(u_{0}\right)<t\right)=0 \quad \text { and } \quad \mathbb{E} \int_{0}^{t}\left(|u|_{H^{1}\left(\mathbb{R}^{n}\right)}^{2}+|u|_{L^{2 \sigma+2}\left(\mathbb{R}^{n}\right)}^{4 \sigma+2}\right) d s<\infty,
$$

then

$$
\mathbb{E}(V(u(t))) \leq \mathbb{E}\left(V\left(u_{0}\right)\right)+\left(4 \mathbb{E}\left(G\left(u_{0}\right)\right)+c_{\Phi}^{1}\right) t+\left(8 \mathbb{E}\left(H\left(u_{0}\right)\right)+c_{\Phi}^{2}\right) t^{2}+c_{\Phi}^{3} t^{3}
$$

where $c_{\Phi}^{1}, c_{\Phi}^{2}, c_{\Phi}^{3}$ depend only on $\Phi$. Since the left hand side is always positive, this is impossible if the polynomial - which only depends on $u_{0}$ - on the right hand side takes negative values. Hence for those values of $u_{0},(4.8)$ cannot hold for $t$ larger than the second root of the polynomial in the right hand side of (4.9). We then consider the deterministic problem :

$$
\left\{\begin{array}{l}
i \frac{\partial U}{\partial t}-\left(\Delta U+|U|^{2 \sigma} U\right)=\frac{\partial f}{\partial t}, \\
U(0)=u_{0}
\end{array}\right.
$$

and denote its solution - when it exists - by $U\left(u_{0}, f, \cdot\right)$. For $\tilde{t}>0$, we construct a function $f$ in a conveniently chosen space such that if $U\left(u_{0}, f, \tilde{t}\right)$ replaces $u_{0}$ in (4.9), then the polynomial in the right hand side takes negative values before a fixed time $\bar{t}>0$. The mapping $f \mapsto U\left(u_{0}, f, t\right)$ is continuous into $H^{1}\left(\mathbb{R}^{n}\right)$ and lower semicontinuous into $\Sigma$. This implies that for $g$ close to $f$, the polynomial in (4.9) with $u_{0}$ replaced by $U\left(u_{0}, g, \tilde{t}\right)$ will also take negative values before $\bar{t}$. Since the solution of (3.3) is given by $u(t)=U\left(u_{0}, W, t\right)$ and $W$ is an irreducible process, we deduce that

$$
V(u(\tilde{t}))+\left(4 G(u(\tilde{t}))+c_{\Phi}^{2}\right) t+8 H(u(\tilde{t})) t^{2}+c_{\Phi}^{1} t^{3}
$$

takes negative values for a fixed $t$ between 0 and $\bar{t}$ with a positive probability. In summary, we prove that for arbitrary $\tilde{t}, \bar{t}$ and $u_{0}$, the solution of (3.3) satisfies at time $\tilde{t}$ the conditions to ensure the blow up in the sense of (4.6) or (4.7) before $\tilde{t}+\bar{t}$, which gives the conclusion of Theorem 4.1 since $\tilde{t}$ and $\bar{t}$ may be taken arbitrarily small. The arguments of the proof of Theorem 4.2 consist in showing that under the additional assumptions, one cannot have simultaneously $\mathbb{P}\left(\tau^{*}\left(u_{0}\right)<t\right)=0$ and (4.7), so that (4.6) always happens. This is obtained thanks to a complicated and technical estimate. In fact, working in this way requires a lot of smoothness assumptions and instead of constructing a forcing term $\frac{\partial f}{\partial t}$, we construct $z$ such that the solution of $i \frac{\partial V}{\partial t}-\left(\Delta V+|V+z|^{2 \sigma}(V+z)\right)=0$ goes from $u_{0}$ to a prescribed value. The function $z$ is formally associated to $f$ by the equation $i \frac{\partial z}{\partial t}-\Delta z=\frac{\partial f}{\partial t}$. The detailed proofs are given in [4]. 


\section{Numerical simulations}

Numerical experiment is a powerful tool to study the qualitative behavior of an evolutionary system. We present here some simulations on the stochastic NLS equation with additive or multiplicative noise. We wish in particular to give informations on the influence of the noise on blow-up for a multiplicative or a non smooth noise, since our theoretical results do not cover these cases. Our numerical scheme is based on finite differences in time and space. It is built to preserve essential properties of the NLS equation: when no noise is taken into account, the discrete solution has constant $L^{2}$ norm and energy $H$. We show results in space dimension one, higher dimensional simulations are now being performed and will be presented elsewhere. It is not possible to discretize an equation on the whole real line and, as it is usual, we restrict our attention to a bounded interval $[0, L]$ and impose Neumann boundary conditions. Given a time step $\Delta t$ and a mesh size $\Delta x=\frac{L}{N}$, we approximate $u(n \Delta t, j \Delta x), n \in \mathbb{N}, j \in\{0, \cdots, N\}$ by $u_{j}^{n}$ defined by

$$
\begin{aligned}
\frac{i}{\Delta t}\left(u_{j}^{n+1}-u_{j}^{n}\right)+\frac{1}{(\Delta x)^{2}}( & \left.u_{j+1}^{n+1 / 2}-2 u_{j}^{n+1 / 2}+u_{j-1}^{n+1 / 2}\right) \\
& +\frac{1}{(\sigma+1)} \frac{\left|u_{j}^{n+1}\right|^{2 \sigma+2}-\left|u_{j}^{n}\right|^{2 \sigma+2}}{\left|u_{j}^{n+1}\right|^{2}-\left|u_{j}^{n}\right|^{2}} u_{j}^{n+1 / 2}=f_{j}^{n+1 / 2},
\end{aligned}
$$

where

$$
f_{j}^{n+1 / 2}= \begin{cases}\varepsilon \frac{\chi_{j}^{n}}{\sqrt{\Delta t} \sqrt{\Delta x}} u_{j}^{n+1 / 2} & \text { (multiplicative noise) } \\ \varepsilon \frac{\chi_{j}^{n}}{\sqrt{\Delta t} \sqrt{\Delta x}} & \text { (additive noise) },\end{cases}
$$

$\left(\chi_{j}^{n}\right)_{j, n}$ is a sequence of independent real valued $\mathcal{N}(0,1)$ random variables and $u_{j}^{n+1 / 2}=\frac{1}{2}\left(u_{j}^{n}+u_{j}^{n+1}\right)$. It may easily be seen that in the multiplicative case, the $L^{2}$ norm is conserved. At each time step, we use a fixed point iteration to find $\left(u_{j}^{n+1}\right)_{j=0, \cdots, J}$. Simulations of the equation have been performed in both additive and multiplicative cases with several initial data and various realizations of the noise. Given a threshold, $S$, we decide that a solution has blown up if its maximum amplitude is larger than $S$. We have checked that this is a good criterion if $S$ is larger than two or three times the amplitude of the initial data. For an initial data $u_{0}$ and a particular form of the equation $(\sigma=2$ or 3 , additive noise or multiplicative), we compute a large number $N$ of realizations. For each realization, we find an estimate of the blow-up time $\tau^{*}$ by taking the first instant when the solution reaches the threshold $S$. We then define $N(t)$ as the number of computed trajectories for which $\tau^{*}$ is greater than $t$ and plot the evolution of $N(t) / N$. As expected, we have found that with an additive noise with critical $(\sigma=2)$ or supercritical $(\sigma=3)$ nonlinearity, $N(t) / N$ is always less than one. However, for $t$ small, we had to simulate more than 30,000 trajectories before being able to see one blow-up before $t$. This shows that $1-N(t) / N$ is very small for $t$ small. Moreover, we have observed that $\frac{N(t)}{N} \rightarrow 0$ when $t \rightarrow+\infty$ so that we may conjecture that solutions blow up at some time with probability one.

In figure 1, we present the same experiment, in the case of a multiplicative noise and a supercritical nonlinearity $(\sigma=3)$ for two kinds of initial data : the first one 
corresponds to a blowing up deterministic solution ; the second one would give a global deterministic solution. We obtain in both cases exactly the same behavior as for an additive noise.

In figure 2 are plotted the results obtained with a critical nonlinearity $(\sigma=2)$. We then see that for an initial data giving rise to a blowing up deterministic solution, we have $\frac{N(t)}{N} \rightarrow 0$ as $t \rightarrow+\infty$ again. However, for an initial data yielding a global deterministic solution, we have not observed any blowing up stochastic solution. It seems that here the noise does not have the effect described above and is not able to create blow-up. We note that our theoretical argument of section 2 cannot be adapted to the multiplicative noise with a critical nonlinearity.

The simulations presented above use a noise which is not white. Its length and time scales are $\Delta t$ and $\Delta x$ while a white noise would have zero length and time scales. In this sense, in the computations presented above, the noise is smooth and this is the reason why we recover the results of section 3. It is possible that smaller scales of the noise have different effects. Indeed, some physicists believe that a white noise may prevent the blow-up of solutions (see [2]). Also, it can be seen that, in the multiplicative case, the noise has a tendency to delay blow-up. It seems that the noise disturbs the deterministic mechanism yielding to blow up. However, sometimes the noise has a weaker effect and the mechanism can be turned on and energy can go into the small scales. The numerical noise does not affect scales smaller than the mesh size so that afterwards it is too late to prevent blow up. If this scenario is correct, only a space-time white noise with arbitrary small scales could prevent blow up.

In order to investigate this aspect, we have used a local refinement procedure, so that near the blow up point, the noise has very small length and time scales. In figure 3, we have plotted the evolution of the maximum amplitude of one trajectory with a multiplicative noise and a critical nonlinearity. The initial data is chosen in order that the deterministic solution blows up around $t=0.83$. Without refinement, as previously observed, the solution amplitude reaches a high value and blow up occurs. The second curve is obtained with refinement, and we see that apparently, the formation of the singularity is inhibited by the smaller scales of the noise. We have always found the same behavior for many realizations. We conclude that, contrary to a smooth noise, a multiplicative space-time white noise may prevent the blow-up of solutions.

This typical behavior does not seem to happen in the additive case.

\section{Numerical analysis of the scheme}

Our aim in this section is to justify the simulations presented above. As a first step, we consider a time semi-discrete scheme only. Also, since our aim is to compare the semi-discrete solution to the solution of the continuous multiplicative equation given by Theorem 3.1 as the time step goes to zero, we will assume in all the section that $n \leq 3$, and that $\sigma$ satisfies the assumptions leading to global existence in Theorem 3.1, with moreover $\sigma<1$ if $n=3$. Given a time step $\Delta t$, we set

$$
\chi_{n}=\frac{W((n+1) \Delta t)-W(n \Delta t)}{\sqrt{\Delta t}},
$$


where $W$ is the Wiener process with covariance $\Phi \Phi^{*}$ defined in the introduction. In all what follows, we assume that $\Phi$ is a Hilbert-schmidt operator from $L^{2}\left(\mathbb{R}^{n}\right)$ into $H^{s}\left(\mathbb{R}^{n}\right)$ with $s>1+\frac{n}{2}$. Given a $\mathcal{F}_{0}$-measurable initial data $u_{0}$ which is a.s. in $H^{1}\left(\mathbb{R}^{n}\right)$, we wish to define $u$ at time $(n+1) \Delta t$ from $u$ at time $n \Delta t$ by the identity

$$
\frac{i}{\Delta t}\left(u_{n+1}-u_{n}\right)+\Delta u_{n+1 / 2}+\frac{\lambda}{\sigma+1} \frac{\left|u_{n}\right|^{2 \sigma+2}-\left|u_{n+1}\right|^{2 \sigma+2}}{\left|u_{n}\right|^{2}-\left|u_{n+1}\right|^{2}} u_{n+1 / 2}=\frac{\chi_{n}}{\sqrt{\Delta t}} u_{n+1 / 2}
$$

where we have set again, $u_{n+1 / 2}=\frac{1}{2}\left(u_{n}+u_{n+1}\right)$. It is indeed possible to show that given a $\mathcal{F}_{n \Delta t}$-measurable $u_{n}$ which is a.s. in $H^{1}\left(\mathbb{R}^{n}\right)$, there is a $\mathcal{F}_{(n+1) \Delta t}$-measurable random variable $u_{n+1}$ with $\mathbb{P}\left\{u_{n+1} \in H^{1}\left(\mathbb{R}^{n}\right)\right\}=1$ satisfying (6.11). We then fix $T>0$, and define the process $u^{\Delta t}$ - which is adapted thanks to the preceding remark) - as being constant and equal to $u_{n}$ on each interval $[n \Delta t,(n+1) \Delta t)$ with $0 \leq n \leq \frac{T}{\Delta t}$, and with $u_{n}$ and $u_{n+1}$ related by (6.11). Our convergence result is then the following.

Theorem 6.1 The process $u^{\Delta t}$ converges in probability to the solution of (3.3) in $L^{\infty}\left(0, T ; H^{s}\left(\mathbb{R}^{n}\right)\right)$ for any $s<1$.

A compactness method is used to prove Theorem 6.1 - because no Strichartz estimates are available in the semi-discrete case - so that we need an estimate on the $H^{1}$ norm of $u^{\Delta t}$, uniform in $\Delta t$. Fortunately, the deterministic part of the scheme preserves the energy. A major difficulty in treating the stochastic equation is then the lack of Ito formula for the time-discretization. The tricks used to overcome this difficulty are rather involved.

\section{References}

[1] O. Bang, P.L. Christiansen, F. If, K.O. Rasmussen, Temperature effects in a nonlinear model of monolayer Scheibe aggregates, Phys. Rev. E 49, 4627-4636 (1994).

[2] O. Bang, P.L. Christiansen, F. If, K.O. Rasmussen, White Noise in the Two-dimensional Nonlinear Schrödinger Equation, Appl. Anal. 57, 3-15 (1995).

[3] A. De Boundo, A. Debussche, A stochastic nonlinear Schrödinger equation with multiplicative noise, Comm. Math. Phys. 205 161-181 (1999).

[4] A. De Bounrd, A. Debussche, On the effect of a noise on the solutions of the focusing supercritical nonlinear Schrödinger equation, preprint.

[5] A. De Boundo, A. Debussche, A semi-discrete scheme for the stochastic nonlinear Schrödinger equation, in preparation.

[6] A. De Bouard, A. Debussche, The stochastic nonlinear Schrödinger equation in $H^{1}$, preprint. 

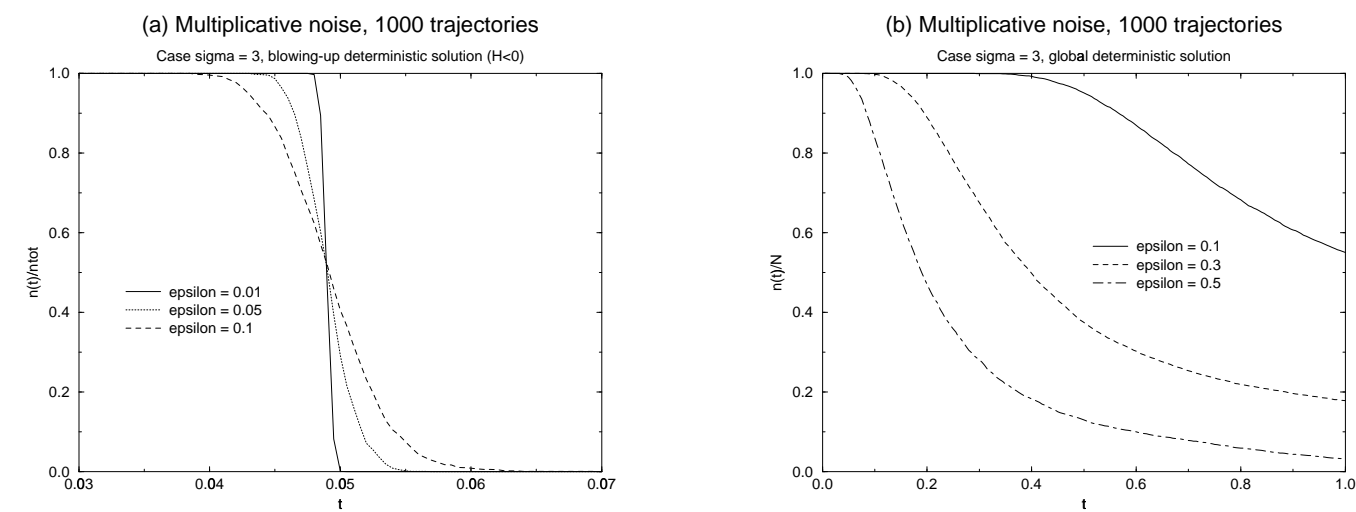

Figure 1: Supercritical case, (a) blowing-up and (b) global deterministic solution, several levels of noise.
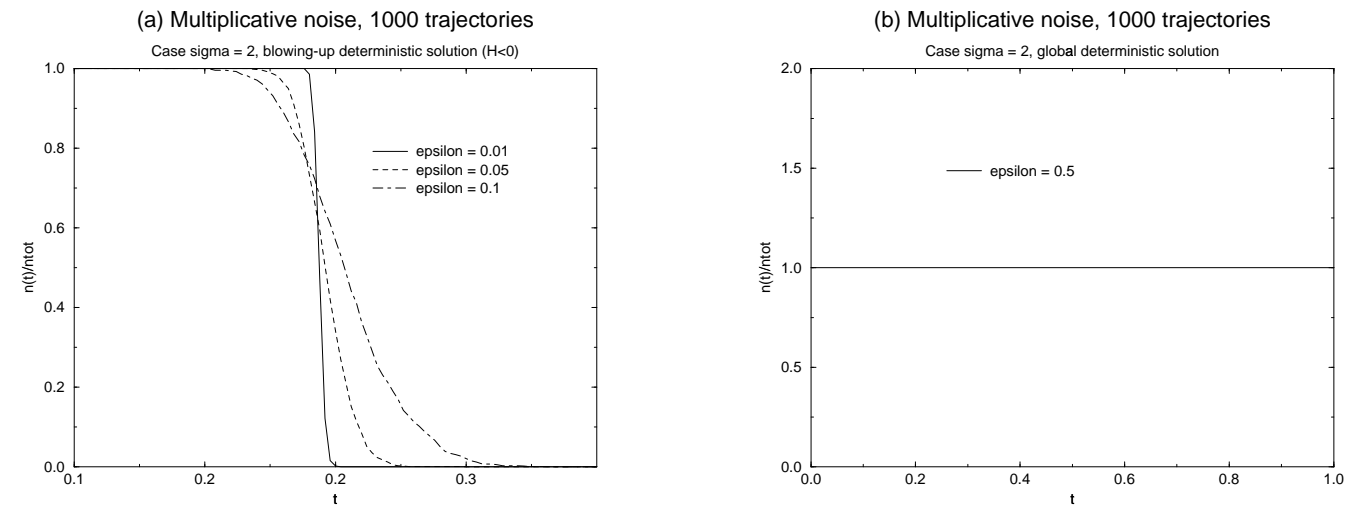

Figure 2: Critical case, (a) blowing-up and (b) global deterministic solution, several levels of noise.

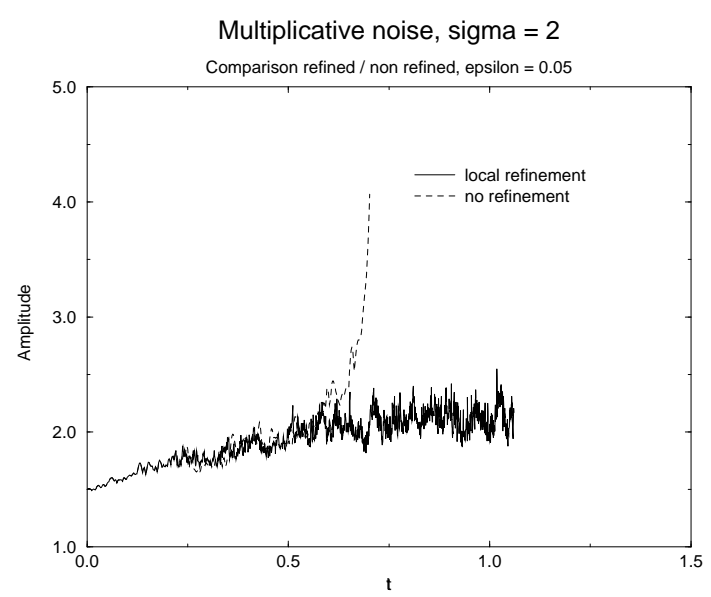

Figure 3: Influence of stochastic refinement on the solution amplitude. 
[7] A. Debussche, L. Di Menza, Numerical simulation of focusing stochastic nonlinear Schrödinger equations, in preparation.

[8] G.E. Falkovich, I. Kolokolov, V. Lebedev, S.K. Turitsyn, Statistics of soliton-bearing systems with additive noise, preprint.

[9] C. Sulem, P.L. Sulem, The Nonlinear Schrödinger Equation, Self-Focusing and Wave Collapse, Appl. Math. Sciences, Springer Verlag, New York (1999).

[10] T. UEDA, W.L. KATH, Dynamics of optical pulses in randomly birefrengent fibers, Physica D 55, 166-181 (1992).

* CNRS et Université PARIS-Sud, URA 760,

BÂT. 425, UNIVERSITÉ DE PARIS-SUd,

91405 ORSAY CEDEX, FRANCE

$\dagger$ Ens de Cachan, Antenne de Bretagne, Campus de Kerlann, Avenue R. Schuman, 35170 BRUZ, FRANCE

arnaud.debussche@bretagne.ens-cachan.fr 